\title{
Impact Damage Sizing with Resonant Frequency Sweep Thermography
}

\author{
by M. Rahammer*, M. Kreutzbruck*
}

* University of Stuttgart, Institut für Kunststofftechnik, Pfaffenwaldring 32, 70569 Stuttgart, Germany, markus.rahammer@ikt.uni-stuttgart.de

\begin{abstract}
Ultrasonic thermography, especially resonant frequency sweep thermography, can utilize local defect resonances to efficiently activate defects in order to increase the defect-selectivity and thus improving the thermal output at the defect. By using a broadband excitation signal in a sweep mode several higher order resonances can be addressed which will then add to the final thermal heat distribution. Due to their convolution the higher order mode shapes are more complex in nature and thus allow for an accurate representation of the actual defect shape. This is proven for a butterfly-shaped impact damage, which is evaluated with several different NDT methods.
\end{abstract}

\section{Introduction}

Ultrasonic excited thermography or vibrothermography is well known for its defect selective, dark-field damage detection capability. It is a well-established NDT technique mainly used for finding cracks in various materials. Next to crack friction, when it comes to polymer-based materials the visco-elastic effect generates significant heat during vibrations. However, it is currently only used with bulky high power equipment. In order to advance this method from its current lab status to a reliable and certified NDT-procedure a reduction in energy consumption combined with improved defect activation and signal processing is necessary. One recent way to achieve this goal is the combination of vibrothermography with local defect resonances (LDR).

The concept of LDR has been thoroughly described in [1, 2]. It is based on the principle that a defect area will have its own resonance frequency, which is usually much higher than the specimen eigenfrequencies due to the lower mass and volume. Flooding a specimen with sound of that specific defect resonance frequency will then result in a strongly vibrating defect, which is by orders of magnitude higher than the negligible vibration mode of the component at this specific frequency. Due to the low mass of the defect area very small energy input is sufficient in order to produce significant heat for thermographic detection as has been demonstrated in [3]. However, the thorough determination of $2 \mathrm{D}$-vibration patterns and size estimation with vibrometry is very time consuming and will always only show the vibration pattern at one particular frequency.

\section{Local defect resonances and wideband excitation}

Obviously, LDR frequencies and mode shapes depend on the geometry of the defect (area) as has been proven in [2] for simple circular flat bottom holes. Later it was demonstrated in [3] that higher order resonance frequencies are able to better reflect the true defect area shape as shown in Fig. 1. The two pictures on the left resemble vibration measurements of a square insert of $20 \times 20 \mathrm{~mm}^{2}$ located at $1.2 \mathrm{~mm}$ depth in a CFRP plate. They were measured with a Laser Doppler Vibrometer, whereas the two corresponding thermograms on the right resemble the same defect and show that the thermal activated area nicely agrees with the region of significant vibration amplitude.
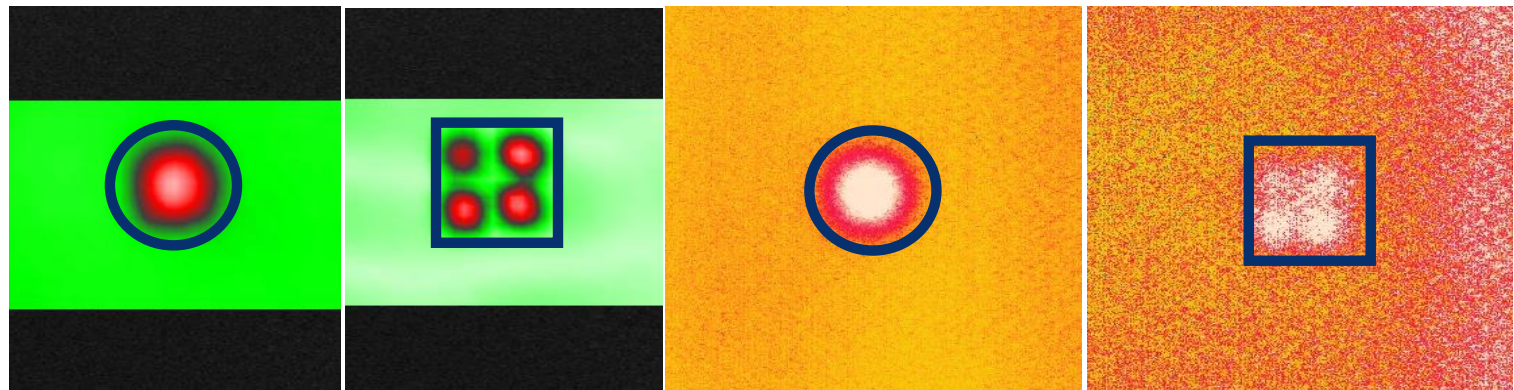

Fig. 1. Qualitative results of vibrometry (left, green-red scaled) and thermography (right, yellow-white scaled) results of a square insert in CFRP excited at fundamental and higher order LDR [2]

The fundamental mode shape of almost every vibrating defect is close to a circular peak. This is shown in the first and corresponding third picture of Fig.1, which have been detected at fundamental frequency excitation. Only when 
using higher order resonance frequencies the true shape of the defect, a square in this case, can be revealed and estimated.

This can be further utilized by using a wideband excitation that includes not only the first order but many higher order resonances. The higher order vibrations all contribute to a mean vibrational state which will roughly resemble the true defect shape as can be seen in Fig. 2 [4].
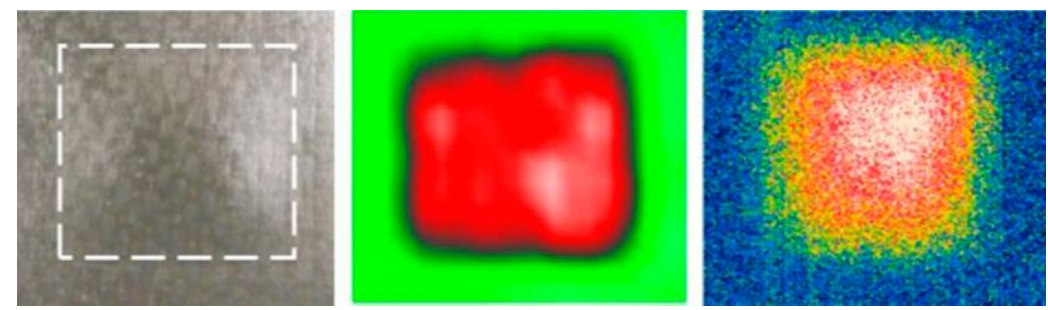

Fig. 2. Photograph (left), Laser Vibrometry RMS result (center) and thermal imaging result (right) of a square insert in CFRP excited with a wideband signal [4]

As these first results have shown, LDR-excited thermography can also be used with a wideband excitation. Very recent advances have merged thermal NDT techniques with a wideband LDR-based excitation. It has been published under the name Resonant Frequency Sweep Thermography (RFST) [5]. It improves standard thermal imaging by slowly $(\sim 60 \mathrm{~s})$ sweeping the excitation signal in order to catch all possible and relevant higher order resonances. Each resonance will contribute to the total thermal pattern with its specific pattern as shown before. By applying a Fourier transformation of the transient thermal data a frequency-selective time-average filtering is applied that significantly reduces the noise. The frequency is selected as such that the sweep time equals the time constant.

This method has been applied successfully to various different applications, for example damage detection in composites [6]. By slowly and repeatedly sweeping the driving frequency source all resonances of potential defects will be fed with enough energy to contribute to the defect heating. The total heat scales with the integral of the amplitudefrequency spectrum, which includes both the characteristic fundamental and the higher order LDR frequency peaks.

\section{Experimental}

As explained, the ensemble of all peaks allows for a reliable estimation of the defect shape with RFST. Especially when it comes to impact damages, one detects their complex distribution of layer-like accumulated intra- and interlaminar damaged areas, each of which giving rise to its own higher order resonances. Comparative measurements with other, established NDT techniques have been performed for an impact damage of $20.1 \mathrm{~J}$ in a $3 \mathrm{~mm}$ woven fabric CFRP which is a double-curved car roof. As can be seen in Fig. 3, the damage is small ( $7 \mathrm{~mm}$ diameter dent) at the front surface, but significant ( $50 \mathrm{~mm}$ long) at the rear.
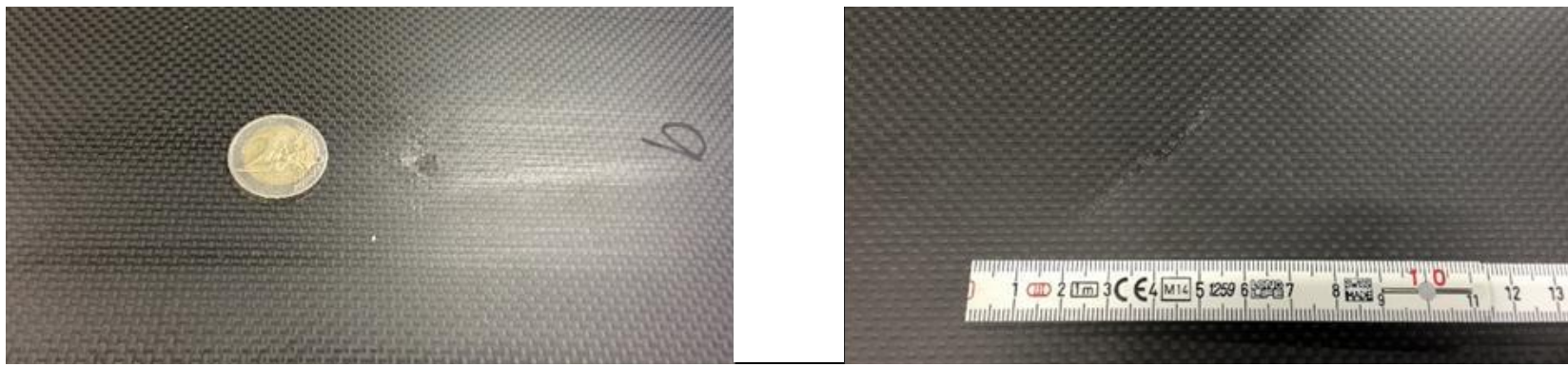

Fig. 3. Impact damage in CFRP: Front surface damage on the left, rear surface on the right.

For comparative measurements computer tomography (CT), Phased Array ultrasonic testing (PA UT), aircoupled ultrasonic testing and optical excitation thermography testing (OTT) were used.

Very high geometric resolution and metrological measurements in 3D are possible with CT. In our case a voxel size of $50 \mu \mathrm{m}$ was used and 1000 steps were made. Special thanks go to Thomas Ullmann at DLR Stuttgart for performing this measurement on their GE phoenix v|tome|x $L 450$ “.

Further high resolution results have been acquired with water-coupled phased array ultrasonic testing, due to the depth focussing technique. For these measurements a COMPAS XL 256 with a SONAXIS 5 MHz probe with $8 \times 8$ elements was used at a step size of $1 \mathrm{~mm}$.

Similarly, air-coupled UT measurements were performed in normal through transmission mode $(200 \mathrm{kHz}$, focused Ultrangroup NCG200-D13-P38 probe, focus $38 \mathrm{~mm}$ ) with a step size of $0.5 \mathrm{~mm}$.

Finally, optical excitation lockin thermography was performed using an edevis $\mathrm{GmbH}$ thermography system with a Flir SC6811 infrared camera (midwave, 25 $\mu \mathrm{m}$ pitch, $18 \mathrm{mK}$ NEDT, $565 \mathrm{~Hz}$ ). 


\section{Results and Discussion}

\subsection{Defect shapes}

All results are assembled for comparison in Fig. 4 starting with RFST in the top left corner. Based on the principles explained above, the approximate shape of the damage can be determined. However, the true size is difficult to measure, as RFST is still a thermal technique and therefore suffers from lateral heat diffusion. This effect is increased by the depth of the delaminations, which typically are close to the rear surface.

a)

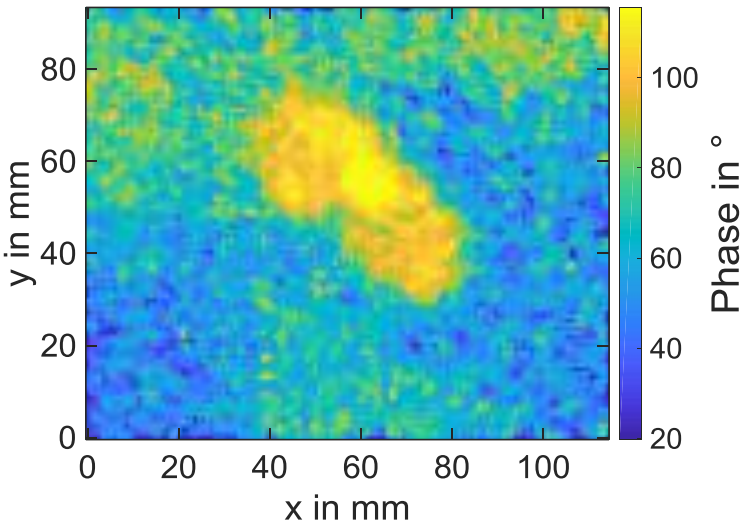

c)

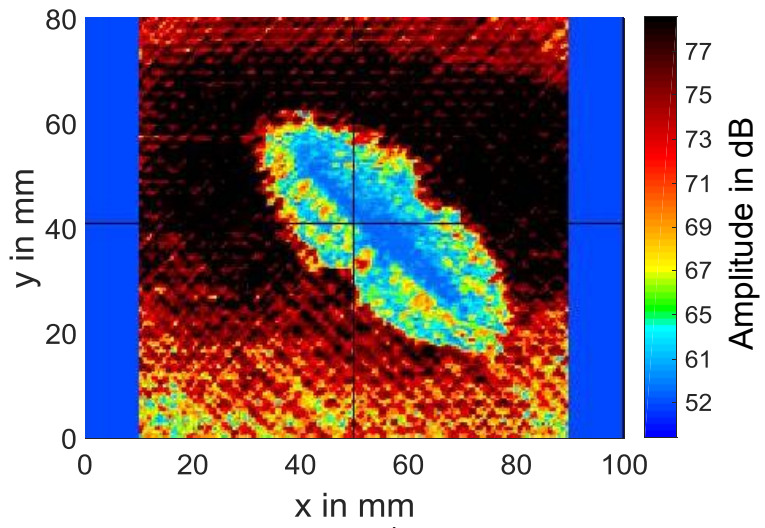

e)

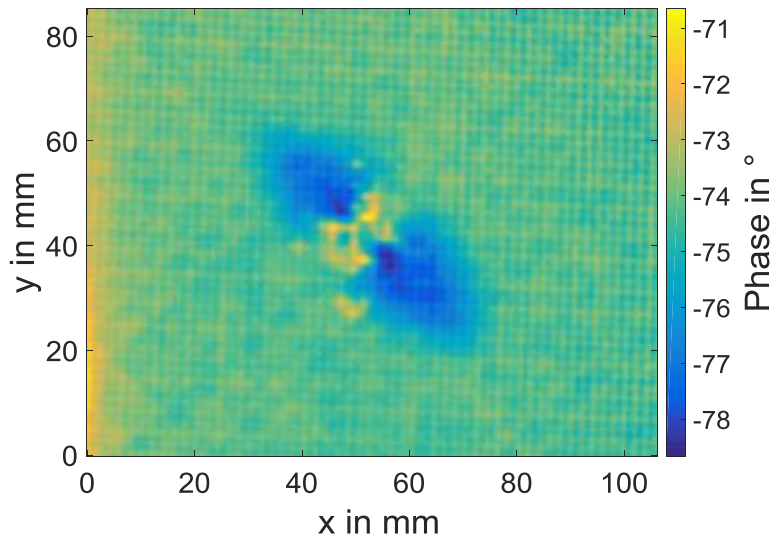

b)

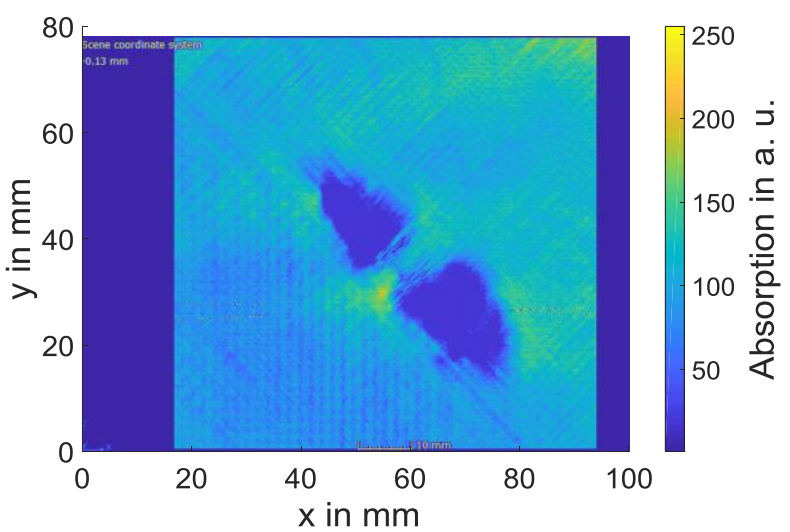

d)

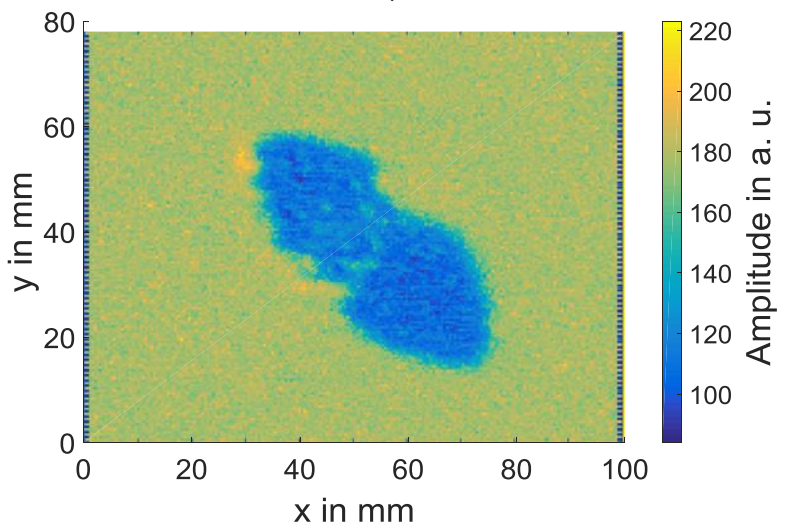

f)

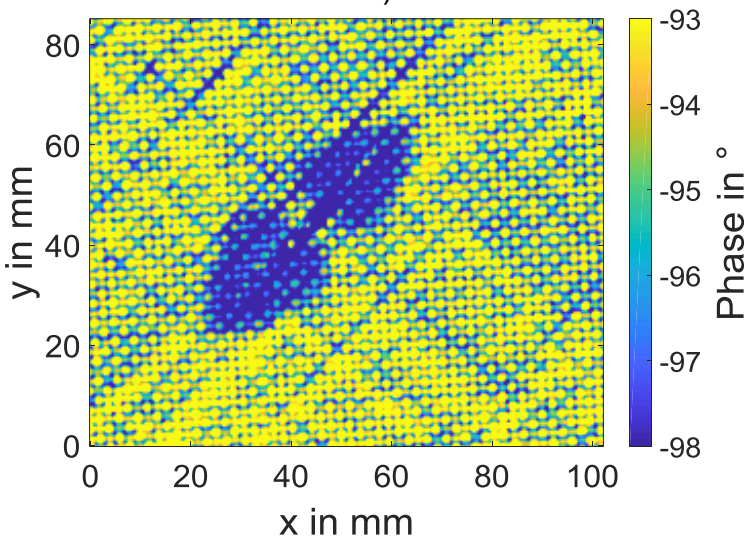

Fig. 4. Comparison of NDT results of an impact damage: A) Resonant Frequency Sweep Thermography, b) computer tomography, c) Phased Array Ultrasonics, d) Air-coupled Ultrasonics, e) optical excitation thermography from the front and f) optical excitation thermography from the rear

CT is often seen as a reference method in the NDT world. For impact damage and delamination evaluation this is less the case. As can be seen in Fig. 4 b) the true perimeter of the delaminations is difficult to determine. The 
delamination's gap becomes infinitesimal small towards the edges and is therefore eventually smaller than the CT resolution which is related to the focus size and step sizes.

In contrast to CT, infinitesimal small gaps at the delamination edges are still detectable with ultrasonics. Any material disbond will result in a reduced transmission of the ultrasonic wave due to the loss of local stiffness. The lateral resolution is similar to PA UT. However, the result is slightly diffuse because of the curved specimen shape not always sitting exactly in at the focus distance of $38 \mathrm{~mm}$

Due to the anisotropic material, the lateral resolution is limited for thermal techniques. Furthermore, a thermal wave is capable of propagating across gaps of up to $10 \mu \mathrm{m}$. Therefore, the delaminations will appear smaller than they are, similar to the CT result. Since impact damage in composites has its largest delamination close to the rear surface, the distance from the front surface to the largest delamination is much higher. Consequently, much lower modulation frequencies are necessary to reach a sufficient depth penetration when testing from the front surface. Low modulation frequencies also have the drawback of increased lateral heat flow in blurriness. This can be seen in the results in Figs. 4 e) and f) which have been acquired from the front with $0.04 \mathrm{~Hz}$ and from the rear with $0.5 \mathrm{~Hz}$.

\subsection{Defect size}

From these two-dimensional data sets the defect contours were determined manually. The area inside the contours was calculated. Due to different lateral resolutions as described above the estimation of the true defect contour has varying preciseness. The error was estimated by repeated measurements. The results for the six measurements shown in Fig. 4 are presented in Table 1.

Table 1. Damage sizing results for an impact damage and various NDT methods

\begin{tabular}{|l|l|}
\hline NDT method & measured impact damage area in $\mathrm{mm}^{2}$ \\
\hline RFST & $860 \pm 21$ \\
\hline CT & $846 \pm 37$ \\
\hline Phased Array UT & $1035 \pm 56$ \\
\hline Air-coupled UT & $1073 \pm 208$ \\
\hline optical Thermography (front) & $885 \pm 53$ \\
\hline optical Thermography (rear) & $854 \pm 29$ \\
\hline
\end{tabular}

As expected, both the ultrasonic techniques provide significantly larger damage areas. This effect has been described with the different signal-defect interaction mechanisms. Both methods present an area of roughly $1050 \mathrm{~mm}^{2}$, but the resolution is much higher for PA UT due to the blurry AC UT result. RFST and optical thermography show very close results because all three are based on thermal heat transfer. The difference between optical thermography front and rear side testing in terms of lateral resolution has been described above, too. Finally, the CT result is close to the thermography methods, although it is based on an entirely different mechanism.

\section{Conclusions}

Ultrasonic thermography, in this case Resonant Frequency Sweep Thermography, is capable of not only detecting impact damages in composite materials, but also sizing them. If the excitation signal included higher order LDR frequencies, their contribution to the RMS vibration results in a good resemblance of the defect shape. The resulting shapes and sizes correspond well with other NDT techniques typically used for composite damage evaluation such as CT or optical excitation thermography. However, ultrasound based methods provide a more precise result of the true total defect size than thermal or $x$-ray techniques. The underestimation of thermal techniques was usually about $20 \%$.

\section{REFERENCES}

[1] Solodov, I., J. Bai, S. Bekgulyan und G. Busse. A local defect resonance to enhance acoustic wave-defect interaction in ultrasonic nondestructive evaluation. In: Applied Physics Letters, 2011, 99(21), 211911.

[2] Solodov, I., J. Bai und G. Busse. Resonant ultrasound spectroscopy of defects. Case study of flat-bottomed holes. In: Journal of Applied Physics, 2013, 113(22), 223512. Doi:10.1063/1.4810926

[3] Solodov, I., M. Rahammer, D. Derusova and G. Busse. Highly-efficient and noncontact vibro-thermography via local defect resonance. In: Quantitative InfraRed Thermography Journal, 2015, 12(1), 98-111

[4] Solodov, I., D. Derusova und M. Rahammer. Thermosonic Chladni figures for defect-selective imaging. In: Ultrasonics, 2015, 60, 1-5

[5] Rahammer, M. und M. Kreutzbruck. Fourier-transform vibrothermography with frequency sweep excitation utilizing local defect resonances. In: NDT \& E International, 2017, 86, 83-88. Doi:10.1016/j.ndteint.2016.11.012

[6] Rahammer, M., I. Solodov, N. Gulnizkij und M. Kreutzbruck. Local Defect Resonance for efficient defect detection in composites. In: ECCM17 - 17th European Conference on Composite Materials, 2016 